\title{
Current status of enthalpy-entropy compensation phenomenon
}

\author{
Satya Priya Moulik ${ }^{1, *}$, Bappaditya Naskar ${ }^{2}$ and Animesh Kumar Rakshit ${ }^{3}$ \\ ${ }^{1}$ Centre for Surface Science, Department of Chemistry, Jadavpur University, Kolkata 700 032, India \\ ${ }^{2}$ Department of Chemistry, Sundarban Hazi Desarat College, University of Calcutta, Pathankhali 743 611, India \\ ${ }^{3}$ Indian Society for Surface Science and Technology, Department of Chemistry, Jadavpur University, Kolkata 700 032, India
}

For similar physical-chemical processes in chemistry and biology, the phenomenon of linear enthalpyentropy compensation (EEC) is a thermodynamic puzzle remaining unexplained for a long time. The basic thermodynamic rules do not rigorously support the EEC phenomenon. In some restricted conditions EEC may appear linear with nonrealistic (i.e. hypothetical) values of the slope (the compensation temperature), and the intercept (the compensation free energy). The compensation temperature $\left(T_{\text {comp }}\right)$ is normally higher than the experimental temperature. Compensation temperature may even become negative, and the related phenomenon is called anti-enthalpy-entropy compensation (AEEC). Negative $T_{\text {comp }}$ is unrealistic. Both EEC and AEEC are not explainable; the derived $T_{\text {comp }}$ and $\Delta G_{\text {comp }}$ (free energy of compensation) of the EEC plot are impractical. The neglect of the Gibbs free energy changes (of similar processes in the EEC plot) makes the phenomenon arbitrary. In a restricted condition (i.e. narrow free energy window range) linear compensation is an assumed solution. In overall consideration, the reported correlations are physicochemically uncertain. The said compensation may arise for both kinetic and equilibrium processes. The manifestations are nearly same. Our demonstration and discussion in this paper pertain to equilibrium processes.

Keywords: Anti-compensation, current status, enthalpyentropy compensation, free energy window.

IT is known for over a century that many similar kinetic and equilibrium processes (both chemical and biochemical) at constant temperature may show linear compensation between enthalpy and entropy whose slope would be different (normally higher) from the experimental temperature. This phenomenon is called 'enthalpy-entropy compensation' (EEC) effect $^{1-5}$. It may be said that no proper explanation of the EEC effect has so far been found, although different kinds of explanations have been put forward. These are: extra-thermodynamic, experimental error related (statistical), solvation effect, presence of microscopic carnot-cycle in the system, etc. In the recent

\footnotetext{
*For correspondence. (e-mail: spmcss@yahoo.com)
}

past, we had revisited the EEC effect, and showed conditions required for its apparent occurrence ${ }^{6-9}$. We showed that a negative or anti-enthalpy-entropy compensation (AEEC) effect might also arise which is reported less in literature $^{10-14}$. However, we would like to mention that several old and recent literature references on EEC can be found in our publications ${ }^{6-8}$ and those of others ${ }^{1-5,15}$. Therefore, elaborate referencing has not been done in this article. We opine that misconception, and non-rigorous uses of thermodynamic parameters have made the effect to appear as a valid finding, masking the real issue. Linear plots normally found in literature are thermodynamically and mathematically not sound. The neglect of the contribution of Gibbs free energy $(G)$ in the manifestation of EEC effect is the main lapse. Planned analysis on similar systems acknowledging the range of Gibbs free energy change $(\Delta G)$ is required for realistic evaluation of both EEC and AEEC manifestations. It is known that EEC is also manifested by kinetic processes in terms of their thermodynamic activation parameters $\left(\Delta G^{\ddagger}, \Delta H^{\ddagger}\right.$ and $\left.\Delta S^{\ddagger}\right)^{4,5}$. The phenomenon for both kinetic and equilibrium processes were earlier discussed together by us ${ }^{6}$. The treatments are basically similar.

Here, we have dealt with the EEC and AEEC of equilibrium processes. Kinetic processes are yet to be known to produce AEEC.

Thermodynamic parameters $(T, \Delta G, \Delta H$ and $\Delta S)$ in framing EEC

Any chemical and biochemical process must be associated with changes in thermodynamic parameters, Gibbs free energy $(\Delta G)$, enthalpy $(\Delta H)$, entropy $(\Delta S)$ and temperature $(T)$. There are well known methods to determine $\Delta G$ and $\Delta H$ (cf. any text book of physical chemistry). Entropy change $\Delta S$ cannot be directly determined but obtained from $\Delta G$ and $\Delta H$ in terms of the following unique and important thermodynamic relation

$$
\Delta H=\Delta G+T \Delta S
$$

At a constant $T$, any thermodynamic process can have distinct measured values of $\Delta G$ and $\Delta H$ which may be used in eq. (1) to derive $\Delta S$. It may be mentioned that 
using $\Delta G$ values at different temperatures, $\Delta H$ values can be derived from van't Hoff equation. This is an indirect way of getting $\Delta H$. By calorimetry, direct determination of $\Delta H$ at a desired temperature can be done with good accuracy $^{16-19}$. For processes (similar or dissimilar) having either the same or very close $\Delta G$ values at a constant $T$, the plot of $\Delta H$ versus $\Delta S$ should yield a straight line (Figure 1) satisfying (eq. (1)). A process done at different temperatures is expected to produce series of straight lines with variable intercepts, $\Delta G_{1}, \Delta G_{2}, \Delta G_{3}$, etc. and slopes, $T_{1}, T_{2}, T_{3}$, etc. (Figure 1 inset). Therefore, temperature has a contribution to the values of other thermodynamic parameters, which is expected. By definition $\Delta S$ is equal to $-(\delta \Delta G / \delta T)$ and experimentally, $\Delta H$ is a function of $T$.

It is stated above that EEC effect arises for similar processes. By similar processes we mean, for example, a kinetic or equilibrium process conducted in different pure or mixed solvents of nearly similar categories. It may also be the solubilization of same category of homologous compounds (like alkanols, amines, ketones, etc.) in a solvent water or otherwise. Dissimilar systems are not considered in the EEC analysis. According to Sharp ${ }^{20}$, EEC is 'a linear relationship between $\Delta H$ and $\Delta S$ for some series of perturbations or changes in experimental variables'. According to $\mathrm{Krug}^{21}$ 'such observed compensations may result for chemical reasons or they may result merely as experimental artifacts'. Fox et al. ${ }^{15}$ opine that the $H / S$ compensation occurs in biomolecular recognition: 'studying $H / S$ compensation can help the still murky-roles of water and dynamics in biomolecular recognition and self-assembly'. Thus different opinions (including completely antagonistic opinions) are found in literature. An unequivocal explanation of the EEC

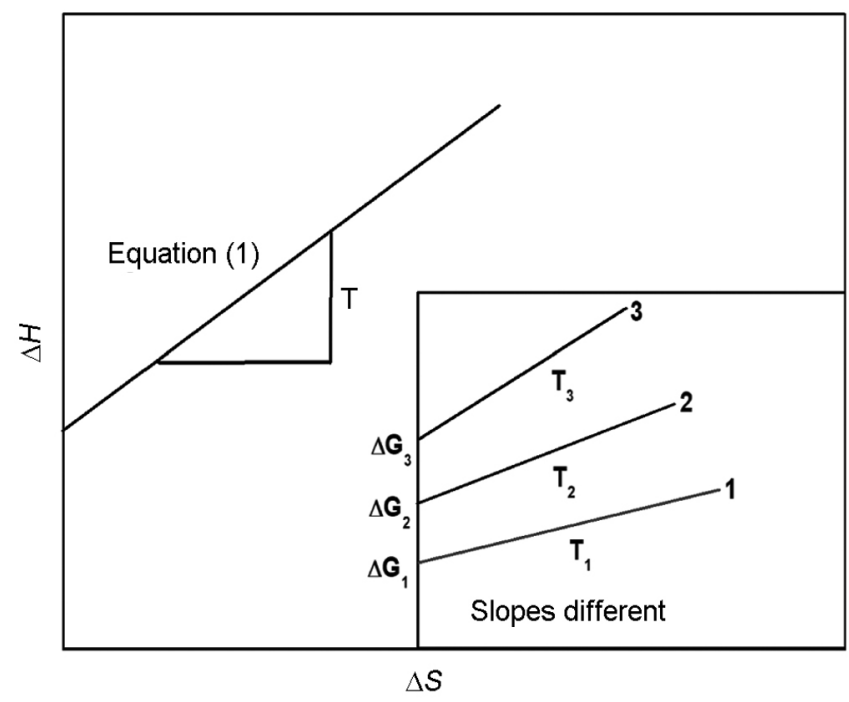

Figure 1. $\Delta H$ versus $\Delta S$ plot according to eq. (1), where $T$ is the experimental temperature (slope), and $\Delta H=\Delta G=$ intercept at $\Delta S=0$. Inset: Same plot at three different temperatures. Gibbs free energy change locations are identified on the ordinate at $\Delta S=0$. process is yet to be made. Literature search finds different categories of similar systems, both kinetic and equilibrium types to manifest $\mathrm{EEC}^{6,7}$. In similar systems, each process has its own $\Delta G, \Delta H$ and $\Delta S$ values at a constant $T$. Their conventional compensation plot is presented in Figure 2, where the slope of EEC line is different from the experimental temperature, $T_{\text {expt }}$ and is called the compensation temperature $T_{\text {comp }}$ which is normally higher than the $T_{\text {expt }}$. According to the reports, EEC may follow the relation

$$
\Delta H=\alpha+\beta \Delta S,
$$

where $\alpha$ and $\beta$ are the intercept and slope respectively, having dimensions of free energy and temperature. Here, $\beta=T_{\text {comp }}$ and $\alpha=\Delta G_{\text {comp }}$ (free energy of compensation); these parameters are obtained only from the compensation plot, which cannot be estimated otherwise. Therefore, $\alpha$ and $\beta$ are thermodynamically non-explainable. In this connection, the rationale of the form of eq. (1), and the thermodynamic nature of the terms $\alpha$ and $\beta$ of eq. (2) have been explained by us in our previous studies ${ }^{6,8}$. Starikov ${ }^{9}$ also dealt with it.

\section{Drawback of the EEC concept}

The conventional linearity of EEC shown in Figure 2 (in terms of eq. (2)) is arbitrary. The course could be haphazard, curved (concave, convex or wavy), and decided by the nature of the process under consideration as well as

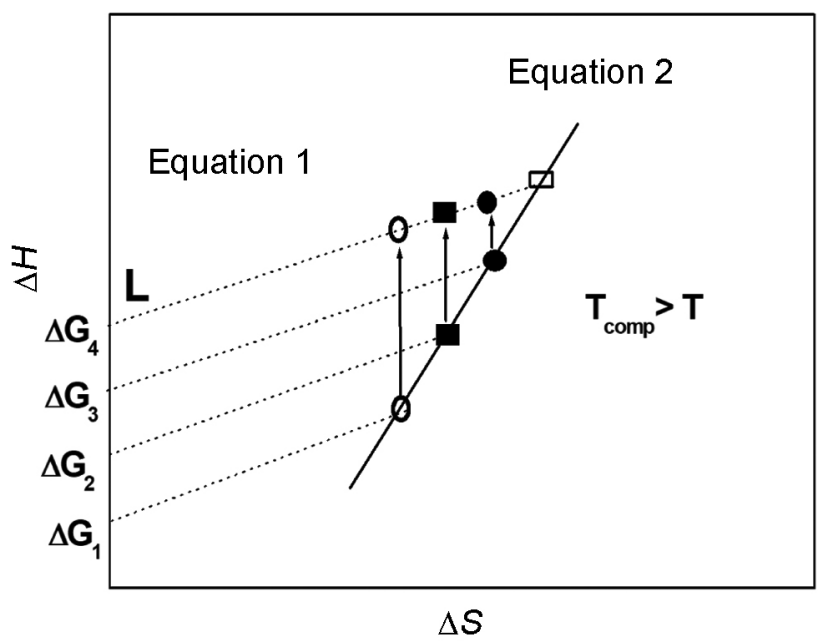

Figure 2. Nature of compensation plot for four similar systems (identified by four symbols) at temperature $T$ (scale, arbitrary). The respective free energy change values are shown on the intercept as defined above. Equation (2) relates the expected linear compensation course and $T_{\text {comp }}>T$. $T_{\text {comp }}=\beta$ and $\alpha=\Delta G_{\text {comp }}$ found from extrapolation at $\Delta S=0$. The free energy range between $\Delta G_{1}$ and $\Delta G_{4}$ is called the free energy window (FEW). For $\Delta G_{1}=\Delta G_{2}=\Delta G_{3}=$ constant $\left(=\Delta G_{4}\right)$, i.e. $\mathrm{FEW}=0$, we get line $L$ following eq. (1) (not a compensation line: slope $\left.=T_{\text {expt }}\right)$ as described in the text. 
the number of similar processes considered in the demonstration, i.e. the range of $\Delta G$ values found or the FEW. These features have been ignored by earlier researchers, where their aim had only been on linearity of EEC which of course resulted from analytical and experimental limitations. The neglect of the importance of $\Delta G$ in the treatment has paid a negative dividend to the results. It is to be mentioned here that Sharp ${ }^{20}$ and Cooper et al. ${ }^{22}$ considered requirement of much lower magnitudes of $\Delta G$ than $\Delta H$ for EEC manifestation. How the width of FEW (irrespective of the magnitudes of the constituent $\Delta G \mathrm{~s}$ ) may control the EEC plot has been recently demonstrated by us $^{8}$. For different similar processes (say four), there should be four $\Delta G \mathrm{~s}$, viz. $\Delta G_{1}, \Delta G_{2}, \Delta G_{3}$ and $\Delta G_{4}$. There will be also four different associated $\Delta H \mathrm{~s}\left(\Delta H_{1}, \Delta H_{2}, \Delta H_{3}\right.$ and $\left.\Delta H_{4}\right)$, and four different $\Delta S \mathrm{~s}\left(\Delta S_{1}, \Delta S_{2}, \Delta S_{3}\right.$ and $\left.\Delta S_{4}\right)$. Plot of each set (say $L$ ) should produce a linear line of intercept, $\Delta G_{1}$, slope, $T$, and the apex in two-dimensional space determined by $\left(\Delta H_{1}, \Delta S_{1}\right)$. Thus, the four sets of results ought to produce four parallel straight lines in the enthalpy-entropy plots. The spread of ' $\Delta G_{1}, \Delta G_{2}, \Delta G_{3}$ and $\Delta G_{4}$ ' is called the FEW.

The above discussed way of plotting is presented for the first time in detail by us. It is obvious that if $\mathrm{FEW}=0$, then the plot in Figure 2 becomes equivalent to the plot in Figure 1 (i.e. a single straight line) following eq. (1) with the slope $=T_{\text {expt }}$. The line $L$ in Figure 2 represents this situation where $\Delta G_{1}=\Delta G_{2}=\Delta G_{3}=$ constant $\left(=\Delta G_{4}\right)$. In such a condition the compensation process does not arise. It is then obvious that depending on the width of FEW the nature of the EEC plot should

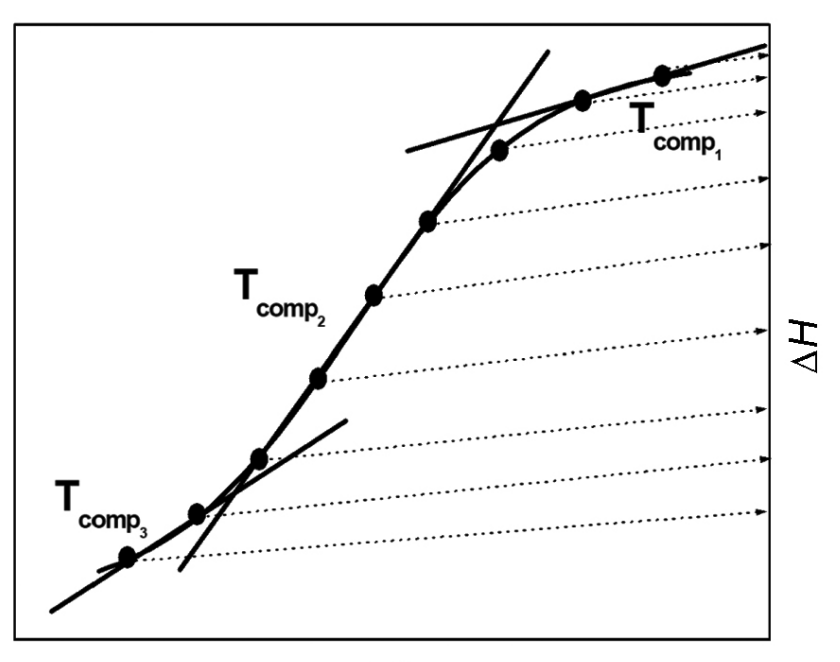

$\Delta S$

Figure 3. A sigmoidal EEC course plotted in arbitrary scale. Short FEW regions can be considered linear. Three arbitrary sections are presented which produce three compensation temperatures. The tangent at each point on the curve is the corresponding $T_{\text {comp }}$ value. The arrow heads on the $Y$-axis correspond to $\Delta G$ values at $\Delta S=0$ of several arbitrary similar processes. change (the illustrated linearity is not though guaranteed). In a narrow FEW range, the EEC plot is closely equivalent to the thermodynamic eq. (1).

In Figure 3, the arbitrarily drawn sigmoidal curve shows possibilities of both concave and convex patterns. Here, in short FEW ranges linearity is obvious. Thus, FEW, an important energetic parameter, although mentioned in literature ${ }^{8,20,22}$, was not seriously considered in the past. We have reported a sigmoidal type EEC manifestation (cf. figure $3 d$ in ref. 8) for ligand binding to biomaterials. The demonstrated plot in Figure 2 was first shown by us ${ }^{8}$, wherein contributions of all the thermodynamic parameters are interrelated as a general depiction of EEC manifestation.

In Figure 4, a practical demonstration of EEC courses is presented. There are enough data points in each to distinctly exhibit both concave $(\boldsymbol{a})$ and convex $(\boldsymbol{b})$ nature of plots discussed above. Here, short regions as usual may show linear trends with varied slopes (Figure 3). In Figure 4 ( $\Delta H$ versus $T \Delta S$ dependence), the linear slope is expected to be an unity. Deviation should be accounted for $T_{\text {comp }}=$ slope $\times T$. The values of the intercept and slope shown are obtained assuming linear plots. It has been found that $T_{\text {comp }} \gg T(298.15 \mathrm{~K})$. The intercepts, i.e. $\Delta G_{\text {comp }}$ are also much different from the experimented values. These slope and intercept values are considered to be the values in the hypothetical state of EEC processes defined in our earlier study ${ }^{6}$. Compensation effect produces a state where $T_{\text {comp }}$ and $\Delta G_{\text {comp }}$ are much different and mostly greater than $T_{\text {expt }}=298.15 \mathrm{~K}$ and the average of the free energies of the similar processes that are dealt with. The systems (alkanes and alkanols) under treatment are physico-chemically much different from what they are at the experimented condition. In the hypothetical states, the alkanes and alkanols at high $T_{\text {comp }}$ (555.6 and $475.6 \mathrm{~K}$ respectively) would be in the gaseous phase; the experimented, gas $\leftrightarrow$ liquid equilibrium should be absent. So, we are talking about impractical outputs from practical conditions. This conceptual paradox of EEC was not addressed in the past. Higher $T_{\text {comp }}$ than $T_{\text {expt }}$ means shifting of the thermodynamic state from the actual. It is a state where all the similar processes in view are supposed to show same or nearly same $\Delta G_{\text {comp }}$ (different from the $\Delta G$ values of all the similar processes considered) at $T_{\text {comp }} \gg T_{\text {expt }}$. In practice, the cited results are implausible, and cannot be error related. Hence, we may conclude that in EEC cases as $T_{\text {comp }}$ is always greater than $T_{\text {expt }}$, they are without a convincing thermodynamic basis.

The above plots are constituted of many systematic experimental points which are normally not available in literature. Usually limited experimented points are displayed with their errors, and apparent linearity is demonstrated as EEC. So the plot becomes a matter of convenience; the produced $T_{\text {comp }}$ and $\Delta G_{\text {comp }}$ are only reported without analysis. Therefore, EEC is a doubtful proposition. 

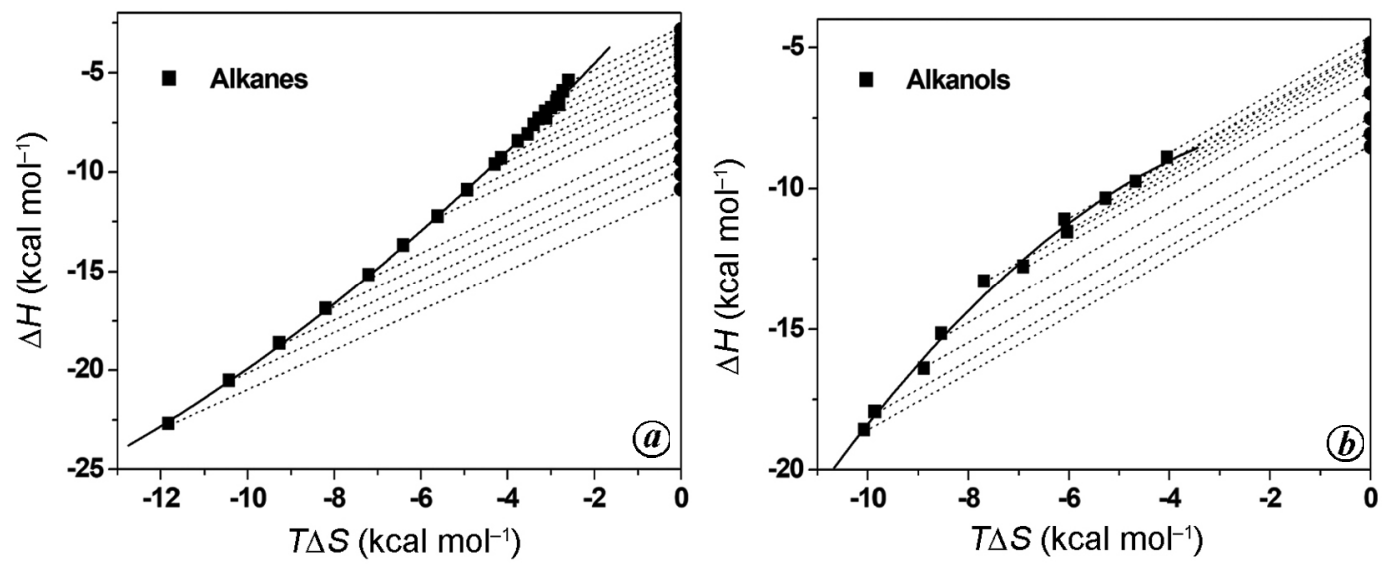

Figure 4. $\Delta H$ versus $T \Delta S$ plots of solvation of vapours of $(\boldsymbol{a})$ alkanes and $(\boldsymbol{b})$ alkanols condensing into their own liquids at $298.15 \mathrm{~K}$ (ref. 29). $\boldsymbol{a}$, Curve with symbols: second order polynomial fit (Regression: 0.995); linear fit slope =1.867; $T_{\text {comp }}=556.6 \mathrm{~K} . \boldsymbol{b}$, Curve with symbols: second order polynomial fit (Regression: 0.993); linear fit slope $=1.595$; $T_{\text {comp }}=475.6 \mathrm{~K}$. Expected linear slopes are greater than unity meaning $T_{\text {comp }}>T_{\text {expt }}$ which are much higher than $298.15 \mathrm{~K}$ as shown above.

\section{Some recent opinions on EEC}

Several recent opinions on EEC manifestations are discussed in the present context. Referring the studies on proteins, protein-ligand interactions, and protein-nucleic acid interactions, Khrapunov ${ }^{23}$ concluded that 'Enthalpyentropy compensation (EEC) is mainly the trivial consequence of the basic thermodynamic laws'; also 'apparent correlation between $H$ and TS can arise because the measured values of $G$ tend to occupy a restricted range while $\Delta H$ and $\Delta S$ can vary over a much wider range - a phenomenon sometimes termed the 'window effect'. Nearly constant free energy change and varied enthalpy and entropy changes of biological binding, and interacting systems are also recently reported in detail by Dragan et $a .^{24}$. They have attempted to explain the constancy of free energy from structural changes, and solvationdesolvation related mechanisms.

In the previous section, we have shown that for a constant FEW or FEW in a narrow range, the compensation eq. (2) is not valid; the results must follow the wellestablished thermodynamic eq. (1). Thus, the findings of Khrapunov $^{23}$ and Dragan et al. ${ }^{24}$ do not throw any new light. The thermodynamics of the systems they have presented do not qualify to be of EEC status. There the slopes of the plots were expected to be $\approx T_{\text {expp. }}$. It may also be mentioned here that Khakhel and Romashko ${ }^{25}$ in a recent paper discussed the compensation phenomenon in relation to pyrene excimer formation process. They explained the phenomenon by using the change in 'phase volume' of the systems. However, it should be noted that in thermodynamics, phase volume is taken to be constant and, therefore, this idea is not contributing any new thermodynamic input in the EEC process.

Vázquez-Tato et al. ${ }^{26}$ published a paper on compensation temperature for micellization of surfactants collect- ing ITC (isothermal titration calorimetry) data of systems at different temperatures. The accepted compensation eq. (2) (presented herein) deals with measurements of similar systems at a constant temperature, and the enthalpyentropy plot produces a slope which differs from the experimental temperature, and it is termed the compensation temperature $\left(T_{\text {comp }}\right)$. The eq. (1) presented by Vazquez-Tato et al. ${ }^{27}: \Delta H=\Delta H_{c}+T_{c} \Delta S$ (intercept stands for 'solute-solute interaction'; slope stands for 'solutesolvent interaction') is not compatible with our requirements. The proposed equation by Chen et $a .^{27}$ too is entirely different from EEC relation. It is also thermodynamically incorrect. Obviously, its use in the literature is also quite limited.

\section{Anti-enthalpy-entropy compensation}

Normally, for different processes we find that enthalpy increases with entropy, the $\Delta H-\Delta S$ plots come out with positive slopes, and $T_{\text {comp }}>T_{\text {expt }}$. For inverse relation between $\Delta H$ and $\Delta S$, the EEC slope becomes reverse, i.e. negative as presented in Figure $5 a$. Linearity may not be observed here also like EEC, as reported in our recent publications $^{8,9}$. In a narrow range of FEW, the results appear to be linear. Anti-enthalpy-entropy compensation (AEEC) is also reported less in literature ${ }^{10-12}$ for gas phase molecular association, substitution, etc. Micelle formation of homologous series of amphiphiles ${ }^{7}$ may also witness AEEC phenomenon. Realistic nonlinear plots are shown in Figure $5 b$ for micellization of ionic liquids BAILs (biamphiphilic ionic liquids) ${ }^{18}$ and AAILs (amino acid-derived ionic liquids) ${ }^{28}$. In AEEC, $T_{\text {comp }}$ is negative, and lower than $T_{\text {expt }}$ leading to a hypothetical state of bigger puzzle, i.e. considerable absurdity for $\Delta G_{\text {comp }}$ (i.e. the intercept) can even become positive (Figures $1 a, 3$ and $5 b$ ). 

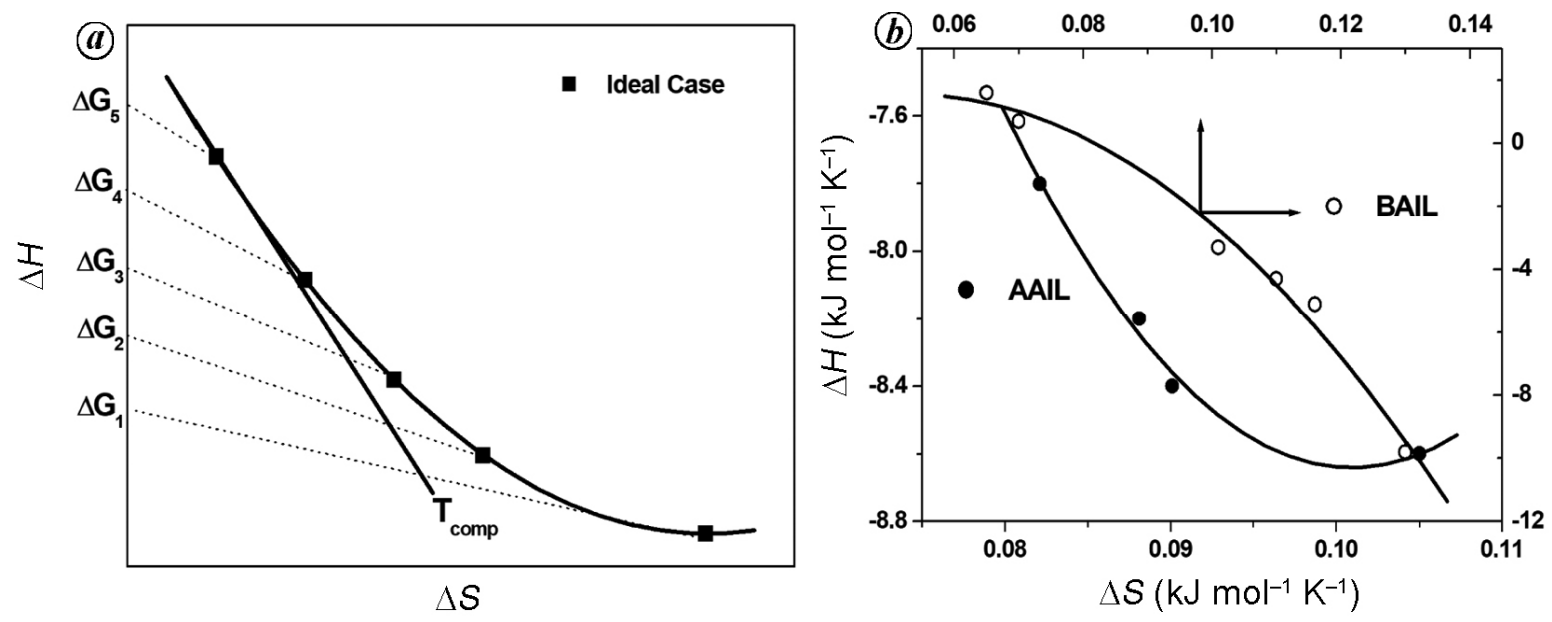

Figure 5. Nature of AEEC plots are schematic and experimental. $\boldsymbol{a}$, Schematic presentation of nonlinear AEEC to make it general. Linearity may be observed for a narrow FEW as presented. $\boldsymbol{b}$, Nonlinear AEEC nature found from ITC measurements for micellization of ionic liquids BAILs ${ }^{18}$ and $\mathrm{AAILs}^{28}$. FEW $=-18.3$ to $-48.7 \mathrm{~kJ} \mathrm{~mol}^{-1}$ for BAILs and -32.7 to $-40.4 \mathrm{~kJ}^{-1}$ for AAILs at $308 \mathrm{~K}$ respectively, and -33.5 to $-41.3 \mathrm{~kJ} \mathrm{~mol}^{-1}$ at $318 \mathrm{~K}$. Nonlinearities found in the plot although FEW values are narrow in AAILs and wide in BAILs.

As an association process reverts to dissociation; a micellization process reverts to demicellization, etc. producing reverse $\Delta H-\Delta S$ correlations.

\section{Conclusion}

EEC is widely studied for many kinds of chemical and biochemical processes but the origin of the phenomenon is still unsolved. Various reasons and explanations have been put forward with the progress of time, but without a pragmatic solution. In EEC, we deal with experimentally determined thermodynamic parameters but talk about something extra-thermodynamic. In this study, eq. (1) has four thermodynamic parameters $(\Delta G, \Delta H, \Delta S$ and $T)$ of which $\Delta G$ has so far been given less or no cognizance in framing the EEC correlation. In our earlier study, and also herein, we have considered the importance of FEW in the explanation, showing that in relation to eq. (1), the EEC plot (eq. (2)) is thermodynamically unsound, and the so called compensation temperature $\left(T_{\text {comp }}\right)$ is a nonexplainable quantity. The intercept of eq. (2) at $\Delta S=0$ has a dimension of free energy $\left(\Delta G_{\text {comp }}\right)$. It also needs an explanation. These two restrictions arising out of experimental measurements strongly raise questions on the manifesting features of EEC. It is a FEW dependent $\Delta H$ $\Delta S$ correlation. The non-linear (curved) plot in a short range of FEW might appear linear, and has been irrationally taken for granted as the linear EEC line ${ }^{8}$. At $\mathrm{FEW}=0$, eq. (2) $=$ eq. (1) with unique values of the slope $\beta=T_{\text {expt }}$ and $\alpha=\Delta G$ (a constant and measurable quantity and not arbitrary). Thermodynamic findings of similar processes as treated in EEC thus produce non-definable results. Therefore, experimentally determined parameters of similar systems are not thermodynamically permitted to combine to produce non-real results. The EEC plot appears to represent an impractical condition where similar physico-chemical processes, if studied at $T_{\text {comp }}$ would all produce equal free energy changes ${ }^{6}$. Therefore, EEC effectively is an impractical proposition (or concept).

The thermodynamic features of EEC discussed above also equally apply to the anti-compensation effect or AEEC, where $T_{\text {comp }}<T_{\text {expt }}$ creating a bigger puzzle to the compensation issue (Figure $5 \mathrm{~b}$ ). A negative $T_{\text {comp }}$ is an unrealistic result obtained from experimentations performed at ambient temperatures and above. Here also, nature of the $\Delta H$ versus $\Delta S$ plot is controlled by the width of $\mathrm{FEW}^{8,9}$.

In conclusion, we state the following: Similar processes at a constant temperature producing different sets of thermodynamic parameters $(\Delta G, \Delta H$ and $\Delta S)$ are thermodynamically non-correlative. Experimentally determined thermodynamic parameters of similar systems processed in a rational way are unlikely to become extrathermodynamic. The EEC, AEEC and NEEC (noncompensation, weak self-association of molecules in the gas phase $)^{9,10}$ phenomena, thereby do not qualify for critical analysis and explanation. In a narrow FEW range in practice, similar systems may apparently exhibit linear EEC correlation but the observed slopes and intercepts may be much different from expectation; they are hypothetical in nature. In a narrow FEW range, dissimilar systems alone or mixed with similar systems can also manifest EECand AEEC-type behaviour challenging the basic premise of the 'so called' EEC and AEEC phenomena. So both the phenomena are fundamentally unreal, physico-chemically uncertain supporting Cornish-Bowden's opinion, i.e. 'enthalpy-entropy compensation: a phantom phenomenon'. 
1. Cornish-Bowden, A., Enthalpy-entropy compensation: a phantom phenomenon. J. Biosci., 2002, 27, 121-126.

2. Lumry, R. and Rajender, S., Enthalpy-entropy compensation phenomena in water solutions of proteins and small molecules: a ubiquitous property of water. Biopolymers, 1970, 9, 1125-1227.

3. Starikov, E. B. and Norden, B., Enthalpy-entropy compensation: a phantom or something useful? J. Phys. Chem. B, 2007, 111, 14431-14435.

4. Linert, W. and Jameson, R. F., The isokinetic relationship. Chem. Soc. Rev., 1989, 18, 477-505.

5. Liu, L. and Guo, Q., Isokinetic relationship, isoequilibrium relationship, and enthalpy-entropy compensation. Chem. Rev., 2001, 101, 673-696.

6. Pan, A., Biswas, T., Rakshit, A. K. and Moulik, S. P., Enthalpyentropy compensation (EEC) effect: a revisit J. Phys. Chem. B 2015, 119, 15876-15884.

7. Pan, A., Rakshit, A. K. and Moulik, S. P., Micellization thermodynamics and the nature of enthalpy-entropy compensation. Colloid. Surf. A, 2016, 495, 248-254.

8. Pan, A., Kar, T., Rakshit, A. K. and Moulik, S. P., Enthalpyentropy compensation (EEC) effect: decisive role of free energy. J. Phys. Chem. B, 2016, 120, 10531-10539.

9. Starikov, E. B., Entropy-enthalpy compensation and its significance in particular for nanoscale events. J. Appl. Solution Chem. Model, 2013, 2, 126-135.

10. Mandal, B., Rakshit, A. K., Moulik, S. P. and Pan, A., A rational study of the origin and generality of anti-enthalpy-entropy compensation (AEEC) phenomenon. Z. Phys. Chem., 2018, 232, $373-$ 391.

11. Ford, D. M., Enthalpy-entropy compensation is not a general feature of weak association. J. Am. Chem. Soc., 2005, 127, 1616716170.

12. Piguet, C., Enthalpy-entropy correlations as chemical guides to unravel self-assembly processes. Dalton Trans., 2011, 40, 80598071 .

13. Graziano, G., Case study of enthalpy-entropy noncompensation. J. Chem. Phys., 2004, 120, 4467-4471.

14. Dunitz, J. D., Win some, lose some: enthalpy-entropy compensation in weak intermolecular interactions. Chem. Biol., 1995, 2, 709-712.

15. Fox, J. M., Zhao, M., Fink, M. J., Kang, K. and Whitesides, G. M., The molecular origin of enthalpy/entropy compensation in biomolecular recognition. Annu. Rev. Biophys., 2018, 47, 223-250.

16. Chatterjee, A., Moulik, S. P., Sanyal, S. K., Mishra, B. K. and Puri, P. M., Thermodynamics of micelle formation of ionic surfactants: a critical assessment for sodium dodecyl sulfate, cetyl pyridinium chloride and dioctyl sulfosuccinate ( $\mathrm{Na}$ salt) by microcalorimetric, conductometric and tensiometric measurements. J. Phys. Chem. B, 2001, 105, 12823-12831.

17. Moulik, S. P. and Mitra, D., Amphiphile self-aggregation: an attempt to reconcile the agreement-disagreement between the enthalpies of micellization determined by the van't Hoff and calorimetry methods. J. Colloid Interf. Sci., 2009, 337, 569-578.

18. Rao, K. S., Trivedi, T. J. and Kumar, A., Aqueous-biamphiphilic ionic liquid systems: self-assembly and synthesis of gold nanocrystals/microplates. J. Phys. Chem. B., 2012, 116, 14363-14374.

19. Blandamer, M. J., Cullis, P. M. and Gleeson, P. T., Three important calorimetric applications of a classic thermodynamic equation. Chem. Soc. Rev., 2003, 32, 264-267.

20. Sharp, K., Entropy-enthalpy compensation: fact or artifact? Protein Sci., 2001, 10, 661-667.

21. Krug, R. R., Detection of the compensation effect ( $\theta$ rule). Ind. Eng. Chem. Fundamen., 1980, 19, 50-59.

22. Cooper, A. C., Johnson, M., Lakey, J. H. and Nöllmann, M., Heat does not come in different colours: entropy-enthalpy compensation, free energy windows, quantum confinement, pressure perturbation calorimetry, solvation and the multiple causes of heat capacity effects in biomolecular interactions. Biophys. Chem., 2001, 93, 215-230.

23. Khrapunov, S., The enthalpy-entropy compensation phenomenon. Limitations for the use of some basic thermodynamic equations. Curr. Protein Pept. Sci., 2018, 19, 1088-1091.

24. Dragan, A. I., Read, C. M. and Crane-Robinson, C., Enthalpyentropy compensation: the role of solvation. Eur. Biophys. J., 2017, 46, 301-308.

25. Khakhel, O. A. and Romashko, T. P., The origin of extrathermodynamic compensations. Heliyon, 2019, 5, e01839.

26. Vázquez-Tato, M. P., Meijide, F., Seijas, J. A., Fraga, F. and Vázquez Tato, J., Analysis of an old controversy: the compensation temperature for micellization of surfactants. $A d v$. Colloid. Interf. Sci., 2018, 254, 94-98.

27. Chen, L.-J., Lin, S.-Y. and Huang, C.-C., Effect of hydrophobic chain length of surfactants on enthalpy-entropy compensation of micellization. J. Phys. Chem. B, 1998, 102, 4350-4356.

28. Rao, K. S., Singh, T., Trivedi, T. J. and Kumar, A., Aggregation behaviour of amino acid ionic liquid surfactants in aqueous media. J. Phys. Chem. B, 2011, 115, 13847-13853.

29. Ben-Naim, A. and Marcus, Y., Solvation thermodynamics of nonionic solutes. J. Chem. Phys., 1984, 81, 2016-2027.

ACKNOWLEDGEMENTS. S.P.M. acknowledges with appreciation Jadavpur University and Indian National Science Academy, New Delhi for Emeritus Professorship and Emeritus Scientist positions respectively. B.N. acknowledges Centre for Surface Science, Department of Chemistry, Jadavpur University for required facilities. A.K.R. thanks AICTE, New Delhi for a former Emeritus Scientist position.

Received 2 May 2019; revised accepted 16 July 2019

doi: $10.18520 / \mathrm{cs} / \mathrm{v} 117 / \mathrm{i} 8 / 1286-1291$ 